\title{
Left ventricular-arterial coupling relations in the normal human heart
}

\begin{abstract}
This investigation was undertaken to assess left ventricular-arterial coupling relations in the normal human heart under varying loading conditions and inotropic states and thereby to establish whether the working point of the normal human heart is at optimal output or mechanical efficiency under basal hemodynamic conditions. In 22 patients with an atypical chest pain syndrome who had normal coronary arteriograms, left ventricular (LV) pressures, volumes, ejection fractions, and masses at cardiac catheterization, we acquired radionuclide angiograms in duplicate simultaneously with micromanometer LV pressures. These values were derived under control conditions and during methoxamine and nitroprusside infusions with heart rate held constant by right atrial pacing. Seven other patients underwent the same protocol but, in addition, we acquired these parameters during a steady-state, intravenous infusion of dobutamine $(5 \mu \mathrm{g} / \mathrm{kg} / \mathrm{min})$. The interaction of $L V$ chamber elastance $\left(E_{e s}\right)$ and effective arterial elastance $\left(E_{a}\right)$ revealed that the normal human heart was operating at an $E_{e s} / E_{a}$ ratio of 1.62, a stroke work of $76 \pm 31 \mathrm{gm}-\mathrm{m}$, and a mechanical efficiency (stroke work to pressure-volume area ratio [SW/PVA]) of $0.65 \pm 0.10$. With an increase in $L V$ load, the $E_{e s} / E_{a}$ ratio approached 1 $(p<0.01)$, LV stroke work increased $(p<0.01)$, and mechanical efficiency declined $(p<0.01)$. In contrast, during vasodilation, the $E_{e s} / E_{a}$ ratio increased to slightly above $2.0(p<0.01)$, LV stroke work decreased $(p<0.001)$, and mechanical efficiency improved $(p<0.01)$. During the dobutamine infusion, similar observations were made for $E_{e s} / E_{a}$, LV stroke work, and SW/PVA over a similar range of LV loading conditions, but enhanced inotropy improved the energy transfer from the left ventricle to the arterial system at comparable $E_{e s} / E_{a}$ ratios without affecting mechanical efficiency. In conclusion, these data indicate that the normal human heart operates at neither optimal output nor efficiency. The working point, however, more closely approximates maximal mechanical efficiency than maximal LV output, but the normal human heart operates over a narrow range of LV SW values. (AM HEART J 1993;125:1659.)
\end{abstract}

Mark R. Starling, MD Ann Arbor, Mich.

The performance of the left ventricle (LV) cannot be fully comprehended in the normal and diseased heart without understanding the interaction of the LV with the systemic arterial system. Sunagawa et al. ${ }^{1,2}$ have proposed a theoretic model to characterize this interaction. Using the end-systolic pressure-volume relation $\left(E_{e s}\right)$ to define $L V$ chamber elastance and the end-systolic pressure $\left(\mathrm{P}_{\mathrm{es}}\right)$-stroke volume relation to

From the Division of Cardiology, Department of Internal Medicine, the University of Michigan and Veterans Administration Medical Centers.

Supported by National Institutes of Health grant R01-HL36540 from the National Heart, Lung, and Blood Institute, Bethesda, Md., and by the Veterans Administration, Washington, D.C. Dr. Starling is the recipient of a National Institutes of Health Research Career Development Award K04HL01787 from the National Heart, Lung, and Blood Institute, Bethesda, Md.

Received for publication Sept. 8, 1992; accepted Dec. 14, 1992.

Reprint requests: Mark R. Starling, MD, Division of Cardiology, Department of Internal Medicine, Veterans Administration Medical Center, 2215 Fuller Road, Ann Arbor, MI 48105.

Copyright 1993 by Mosby-Year Book, Inc.

$0002 \cdot 8703 / 93 / \$ 1.00+.10 \quad \mathbf{4} / \mathbf{1} / \mathbf{4 5 4 2 4}$ define effective arterial elastance $\left(\mathrm{E}_{\mathrm{a}}\right)$, they proposed that the interaction of $E_{e s}$ and $E_{a}$ would be useful for characterizing $L V$ pump function under varying loading and inotropic conditions. Using an analytic model, Burkhoff and Sagawa ${ }^{3}$ have shown that maximal LV stroke work occurs when the $\mathrm{E}_{\mathrm{es}} / \mathrm{E}_{\mathrm{a}}$ ratio is near unity. The observation that maximal LV output or power occurs when the LV and systemic arterial system are optimally coupled has also been made by others. ${ }^{2,4-11}$

Burkhoff and Sagawa ${ }^{3}$ have also shown that maximal efficiency, defined as the ratio of LV stroke work to myocardial oxygen consumption, occurs when the ratio of $\mathrm{E}_{\mathrm{es}}$ to $\mathrm{E}_{\mathrm{a}}$ is near 2.0. They suggested that for normal physiologic LV pressures, end-diastolic volumes, stroke volumes, and ejection fractions to exist, the normal LV would probably operate closer to maximal efficiency rather than to maximal stroke work. Around what working point of LV output and mechanical efficiency the normal human heart is regulated under basal hemodynamic conditions has 
not been elucidated. Accordingly, this investigation was undertaken to assess the effects of alterations in LV load and inotropy on LV output and mechanical efficiency and thereby to determine whether the working point of the normal human heart is at optimal output or mechanical efficiency under basal hemodynamic conditions.

\section{METHODS}

Patient population. The study population consisted of 29 patients, who were referred for cardiac catheterization to evaluate an atypical chest pain syndrome. There were 23 men and 6 women, with an age range of 33 to 71 years (52 $\pm 10[\mathrm{SD}]$ years). All patients had a normal physical examination, electrocardiogram, chest radiograph, and normal coronary arteries at cardiac catheterization. The cardiac catheterization also demonstrated normal left ventricular pressures, volumes, ejection fractions, and masses. All patients had medications withheld for at least 24 hours. Each patient also provided written informed consent for the investigative protocol on forms approved by the $\mathrm{Hu}$ man Studies Committees at the University of Michigan or Veterans Administration Medical Centers, Ann Arbor, Mich.

Protocol. Following standard cardiac catheterization, 22 patients had radionuclide angiograms acquired in duplicate simultaneously with micromanometer LV pressures under control conditions and during methoxamine or nitroprusside infusions. Heart rate was held constant by right atrial pacing. Seven patients underwent this phase of the protocol and, in addition, they had radionuclide angiograms acquired in duplicate simultaneously with micromanometer LV pressures under control conditions and following methoxamine or nitroprusside infusions during the steady-state, intravenous administration of dobutamine $(5 \mu \mathrm{g} / \mathrm{kg} / \mathrm{min})$.

Hemodynamics. The hemodynamic data acquisitions performed in this laboratory have been described in detail elsewhere. ${ }^{12,13} \mathrm{~A}$ bipolar right atrial pacing catheter was placed in the right atrial appendage to maintain heart rate constant, and a micromanometer catheter was positioned in the $\mathrm{LV}$ to measure $\mathrm{LV}$ and aortic pressures simultaneously with each radionuclide acquisition. These recordings were obtained for 10 to 20 cardiac cycles at the beginning, middle, and end of each radionuclide acquisition. An average $L V$ pressure waveform was obtained and was hand-digitized. ${ }^{14}$ The program developed in our laboratory yields instantaneous LV pressure and the first derivative of LV pressure at a variable sampling frequency from the peak of the $\mathrm{R}$ wave. It also allows for interpolation to provide $L V$ pressure data points that correspond to the midpoint of each radionuclide frame.

Radionuclide angiography. The radionuclide angiographic data acquisitions performed in this laboratory have also been described in detail elsewhere. ${ }^{15}$ Briefly, gated equilibrium radionuclide angiograms were acquired after in vivo red blood cell labeling with technetium- $99 \mathrm{~m}$ for 30 msec frames throughout the cardiac cycle for 250 cardiac cycles. During the midportion of each radionuclide acqui- sition, a $2 \mathrm{ml}$ blood sample was drawn and was later counted for 2 minutes. This time delay was recorded for decay correction. At the end of the protocol, a distance measurement was obtained for attenuation correction. Attenuation-corrected radionuclide $L V$ volumes were then calculated frame-by-frame from background-subtracted hand-drawn region-of-interest LV count data, decay-corrected blood sample counts, and attenuation correction, as previously validated in this laboratory. ${ }^{16}$

Data analysis. Corresponding micromanometer LV pressures and radionuclide LV volumes for each loading condition were plotted to obtain multiple pressure-volume loops for each patient. The maximal pressure/volume ratio from each pressure-volume loop was then subjected to linear regression analysis to obtain a slope $\left(\mathrm{E}_{\mathrm{es}}\right)$ reflecting $\mathrm{LV}$ chamber elastance. Similarly, for each pressure-volume loop, $\mathrm{P}_{\mathrm{es}}$, defined as $\mathrm{LV}$ pressure at the maximum pressure/ volume ratio, was divided by radionuclide $L V$ stroke volume (SV), defined as the difference between the radionuclide maximum $L V$ volume and radionuclide $L V$ volume at $P_{e s}$, to obtain a slope $\left(E_{a}\right)$ reflecting effective arterial elastance. ${ }^{1,2}$ 'The coupling of the $L V$ and systemic arterial system was then expressed as the ratio of $E_{\mathrm{es}} / \mathrm{E}_{\mathrm{a}}$.

The pressure-volume area (PVA) as defined by Suga et al. ${ }^{17}, 18$ was calculated for each $\mathrm{LV}$ loading condition and inotropic state. The PVA was defined as the area encompassed by $E_{\mathrm{es}}$, the diastolic pressure-volume curve, and the systolic portion of each pressure-volume loop. LV stroke work (SW) was obtained by calibrated planimetry of each pressure-volume loop and then by multiplying by 0.0136 to convert from millimeters of mercury per milliliter to grammeters. LV pressure-volume areas are representative of the total mechanical energy available to the LV to perform external work. These areas have the components of external work and potential energy. ${ }^{18}$ The latter can perform external work under the appropriate set of LV loading conditions..$^{19}$ It has also been shown that the LV PVA has a linear relationship with myocardial oxygen consumption. 20,21 Therefore, since the traditional definition of myocardial efficiency is the ratio of external work performed to myocardial oxygen consumption $\left(\mathrm{MVO}_{2}\right)$, we used the LV SW/ PVA relationship in this investigation to reflect the mechanical efficiency of converting the total mechanical energy (PVA) available to the LV to external work (SW). It is important to recognize that, although LV PVA has a linear relationship with $\mathrm{MVO}_{2}$, there is a variable $\mathrm{Y}$-axis offset caused by basal metabolism that is not reflected by PVA. Thus LV mechanical efficiency, as defined in this investigation, is not synonymous with myocardial efficiency.

It has previously been proposed that the position of the end-systolic pressure-volume relation may be quantitated in each heart by determining the volume (V) at a common $\mathrm{P}_{\mathrm{es}}{ }^{22} \mathrm{We}$ quantitated the position of the end-systolic pressure-volume relation at a $\mathrm{P}_{\mathrm{es}}$ of $125 \mathrm{~mm} \mathrm{Hg}$ using the equation: $V_{12,5}=V_{0}+125 / E_{e s}$, where $V_{0}$ is the unstressed volume. This volume was chosen lo approximate the average $P_{e s}$ value during control conditions and so that each $E_{e s}$ would be calculated from pressure/volume ratios that would encompass this value for $P_{e s}$.

Further, we quantitated the effects of changes in LV 

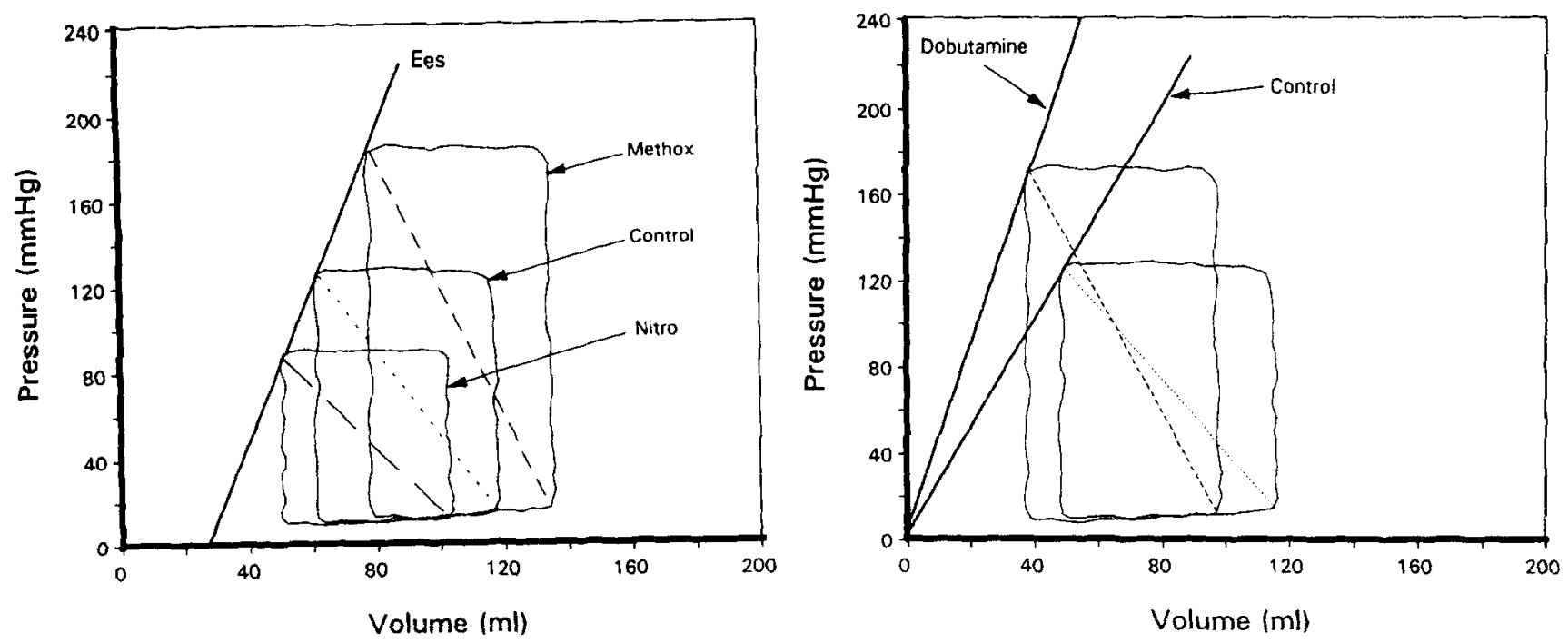

Fig. 1. Left panel, Schematic representation of the effects of altered $L V$ load on effective arterial elastance and the relationship with LV chamber elastance. Right panel, Effects of enhanced inotropy on the relationship between $L V$ chamber elastance and effective arterial elastance. $E_{e s}$, LV chamber elastance; $E_{a}$, effective arterial elastance; Methox, methoxamine; Nitro, nitroprusside.

Table I. Effects of altered load on hemodynamics and left ventricular-arterial coupling

\begin{tabular}{lccccccccc}
\hline & $\begin{array}{c}E_{e s} \\
(\mathrm{~mm} \mathrm{Hg} / \mathrm{ml})\end{array}$ & $\begin{array}{c}V_{o} \\
(\mathrm{ml})\end{array}$ & $\begin{array}{c}P_{\text {es }} \\
(\mathrm{mm} \mathrm{Hg})\end{array}$ & $\begin{array}{c}E D V \\
(\mathrm{ml})\end{array}$ & $\begin{array}{c}S V \\
(\mathrm{ml})\end{array}$ & $\begin{array}{c}E_{a} \\
(\mathrm{~mm} \mathrm{Hg} / \mathrm{ml})\end{array}$ & $E_{\text {es }} / E_{a}$ & $\begin{array}{c}S W_{100} \\
(\mathrm{gm}-\mathrm{m})\end{array}$ & $S W / P V A$ \\
\hline Control & $3.51 \pm 1.26$ & $1 \pm 23$ & $126 \pm 18$ & $99 \pm 39$ & $59 \pm 20$ & $2.32 \pm 0.61$ & $1.62 \pm 0.80$ & $76 \pm 31$ & $0.65 \pm 0.10$ \\
Methoxamine & & & $166 \pm 23 \ddagger$ & $112 \pm 44^{*}$ & $62 \pm 26$ & $3.03 \pm 1.21^{*}$ & $1.31 \pm 0.68^{*}$ & $93 \pm 42^{*}$ & $0.60 \pm 0.11^{*}$ \\
Nitroprusside & & & $92 \pm 16 \ddagger$ & $86 \pm 37 \dagger$ & $57 \pm 21$ & $1.85 \pm 0.70 \dagger$ & $2.26 \pm 1.51 \dagger$ & $58 \pm 25 \dagger$ & $0.72 \pm 0.13^{*}$ \\
\hline
\end{tabular}

$E_{e s}$, Left ventricular chamber elastance; $V_{0}$, unstressed volume; $P_{e s,}$, left ventricular pressure at end systole; $E_{a}$, effeclive vascular elasiance; $E D V$, left ventricular end-diastolic volume; $S V$, left ventricular stroke volume; $S W /$ toln, left ventricular stroke work standardized to a common $V_{d}$ of $100 \mathrm{ml} ; S W / P V A$, left ventricular mechanical efficiency.

${ }^{*} p<0.01, \uparrow p<0.001, \ddagger p<0.0001$ versus control.

end-diastolic volume produced by variations in LV loading conditions and inotropic state on SW.23, 24 This was done by establishing the LVSW at a common diastolic volume $\left(V_{d}\right)$ of $100 \mathrm{ml}$. Finally, the LVSW at a $V_{d}$ of $100 \mathrm{ml}$ was then normalized to the maximal LVSW and was expressed as a percent of the maximal LVSW for comparison across the full range of $\mathrm{E}_{\mathrm{es}} / \mathrm{E}_{\mathrm{a}}$ values.

Statistical analyses. The data are expressed as the mean \pm 1 standard deviation (SD). To determine whether differences occurred between hemodynamic measures, $E_{a}$, $\mathrm{E}_{\mathrm{es}} / \mathrm{E}_{\mathrm{a}}, \mathrm{SW}_{100}$ (LV stroke work standardized to a common $\mathrm{V}_{\mathrm{d}}$ of $100 \mathrm{ml}$ ), and SW/PVA at different LV loading conditions and inotropic states, an analysis of variance with repeat measures or paired $t$ tests were performed. When a significant $F$ statistic was obtained, multiple range tests were used to identify differences. Significant differences were established when a probability value of less than 0.05 was obtained.

\section{RESULTS}

Effects of LV load alterations on ventriculo-arterial coupling relations. The average $L V \mathrm{E}_{\mathrm{es}}$ was $3.51 \pm 1.26$ $\mathrm{mm} \mathrm{Hg} / \mathrm{ml}$. The values ranged between 1.65 and 5.61 $\mathrm{mm} \mathrm{Hg} / \mathrm{ml}$. The average volume-axis intercept $\left(\mathrm{V}_{0}\right)$ for $E_{e s}$ was $1 \pm 23 \mathrm{ml}$. The $V_{n}$ values ranged from -23 to $81 \mathrm{ml}$. The effects of $\mathrm{LV}$ load alterations on hemodynamic parameters and left ventricular-arterial coupling relations are shown in Table I and are schematically demonstrated in Fig. 1. During control conditions, $\mathrm{P}_{\mathrm{es}}$ averaged $126 \pm 18 \mathrm{~mm} \mathrm{Hg}, \mathrm{LV} \mathrm{V}$ ed averaged $99 \pm 39 \mathrm{ml}$, and $\mathrm{LV}$ stroke volume averaged $59 \pm 20 \mathrm{ml}$. From these data, effective arterial elastance was calculated to be $2.32 \pm 0.61 \mathrm{~mm} \mathrm{Hg} /$ $\mathrm{ml}$. Thus, the average effective arterial elastance was less than the average $L V E_{e s}$, yielding an $E_{e s} / E_{a}$ ratio of $1.62 \pm 0.80$. The average LVSW was $76 \pm 31$ $\mathrm{gm}-\mathrm{m}$, and the conversion of total mechanical energy to external work (SW/PVA) averaged $0.65 \pm 0.10$.

Increasing $L V$ load with methoxamine produced the expected effects on left ventricular-arterial coupling relations (Table I). The average $P_{e s}$ increased to $166 \pm 23 \mathrm{~mm} \mathrm{Hg}(p<0.0001$ vs control), as did LV 


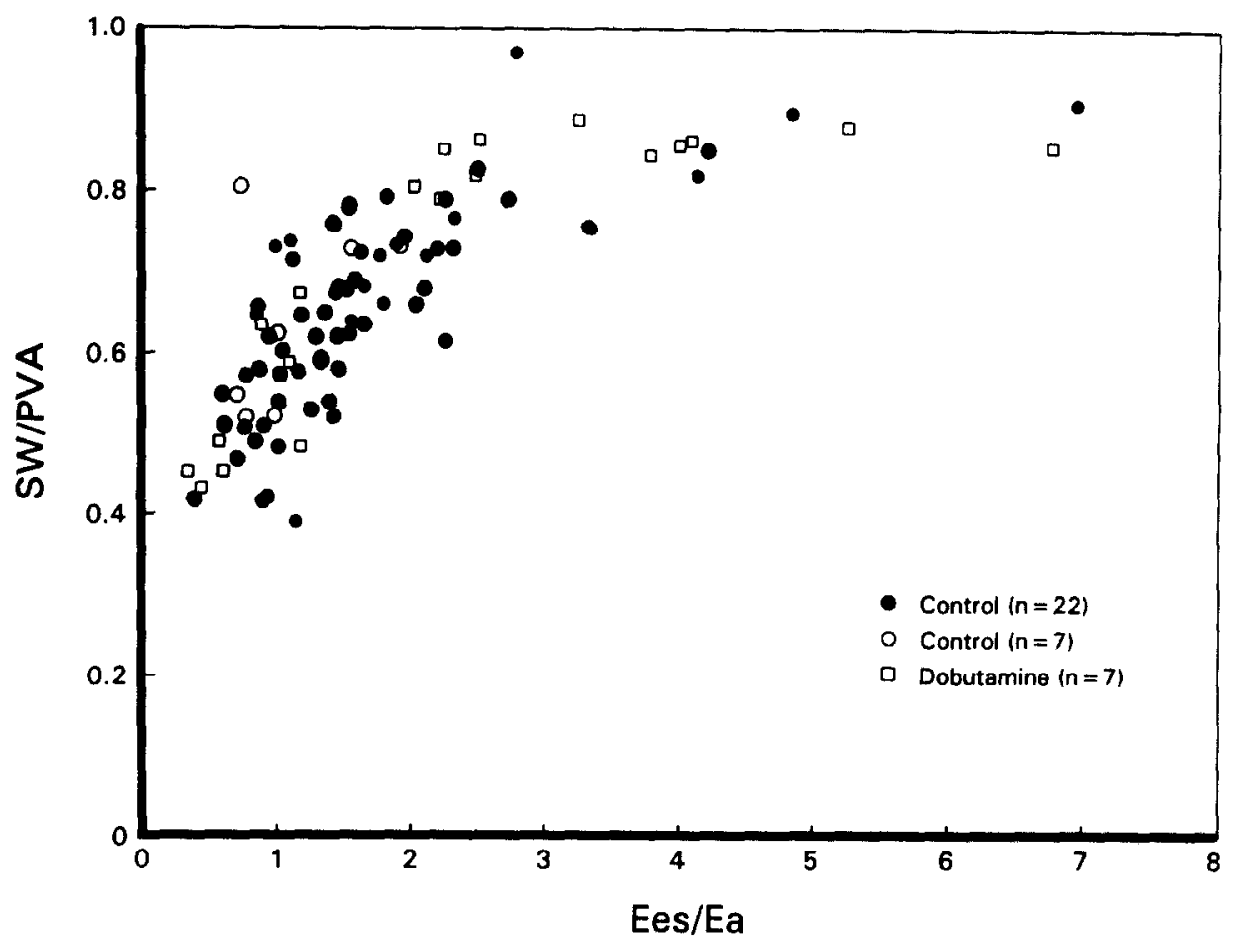

Fig. 2. Relationship between the efficiency of converting the pressure-volume area $(P V A)$ to stroke work $(S W)$ (ordinate) is compared with the left ventricular-arterial coupling relationship $\left(E_{e s} / E_{a}\right)$ (abscissa). From this relationship, the LV appears to operate over a wide range of left ventricular-arterial coupling ratios under basal conditions, while there is a sharp reduction in the efficiency of converting PVA to SW as the $\mathrm{E}_{\mathrm{es}} / \mathrm{E}_{\mathrm{a}}$ ratio declines below 1.0, irrespective of inotropic state.

$\mathrm{V}_{\text {ed }}(112 \pm 44 \mathrm{ml}, p<0.01)$, while $\mathrm{LV}$ stroke volume remained unchanged. Consequently, $\mathrm{E}_{\mathrm{a}}$ increased to $3.03 \pm 1.21 \mathrm{~mm} \mathrm{Hg} / \mathrm{ml}(p<0.01)$. Nevertheless, $\mathrm{E}_{\mathrm{a}}$ remained less than $\mathrm{E}_{\mathrm{es}}$, so that the LV-arterial coupling relationship continued to exceed 1.0 $(1.31 \pm 0.68, p<0.001)$. During this loading condition, LVSW increased to $93 \pm 42$ gm-m $(p<0.01)$, but the mechanical efficiency of the $\mathrm{LV}$ was reduced to $0.60 \pm 0.11(p<0.01)$.

Decreasing LV load with nitroprusside produced opposite effects on these hemodynamic parameters and on LV-arterial coupling relations (Table I). The average $P_{\text {es }}$ was reduced $(92 \pm 16 \mathrm{~mm} \mathrm{Hg}, p<0.0001$ vs control), as was $L V V_{\mathrm{ed}}(86 \pm 37 \mathrm{ml}, p<0.001)$, while $\mathrm{LV}$ stroke volume was unchanged. $\mathrm{E}_{\mathrm{a}}$ was therefore reduced to $1.85 \pm 0.70(p<0.001)$. Consequently, the $\mathrm{E}_{\mathrm{es}} / \mathrm{E}_{\mathrm{a}}$ ratio increased to $2.26 \pm 1.51$ $(p<0.001)$. With the reduction in LV load, LVSW decreased to $58 \pm 25 \mathrm{gm}-\mathrm{m}(p<0.001)$. However, the mechanical efficiency (SW/PVA ratio) of the $I V$ improved to $0.72 \pm 0.13(p<0.01)$.

A plot of the relationship between the efficiency of converting PVA to $\mathrm{SW}$ and $\mathrm{E}_{\mathrm{es}} / \mathrm{E}_{\mathrm{a}}$ for all loading conditions in these 22 patients is shown in Fig. 2. As illustrated in the figure, the mechanical efficiency of the $\mathrm{LV}$ appears to reach a plateau at or just before an $\mathrm{E}_{\mathrm{es}} / \mathrm{E}_{\mathrm{a}}$ value of 2.0. There is a sharp fall in the efficiency of converting PVA to $\mathrm{SW}$ as the $\mathrm{E}_{\mathrm{es}} / \mathrm{E}_{\mathrm{a}}$ ratio declines, particularly to less than 1.0. This is illustrative of the detrimental effects of $\mathrm{LV}$ afterload on mechanical efficiency of the LV. In contrast, as $\mathrm{E}_{\mathrm{es}} / \mathrm{E}_{\mathrm{a}}$ falls toward 1.0, LVSW increases, and as $\mathrm{E}_{\mathrm{es}} / \mathrm{E}_{\mathrm{a}}$ increases, LVSW decreases. A plot of the relationship between normalized LVSW and $\mathrm{E}_{\mathrm{es}} / \mathrm{E}_{\mathrm{a}}$ in Fig. 3 illustrates that, although normalized LVSW increases as the $\mathrm{E}_{\mathrm{es}} / \mathrm{E}_{\mathrm{a}}$ ratio approaches 1.0 , the $L V$ operates over a narrow range of LVSW values. Thus, based on these observations, the LV operates under basal conditions over a broad range of $\mathrm{E}_{\mathrm{es}} / \mathrm{E}_{\mathrm{a}}$ values within $9 \%$ of maximal mechanical efficiency, while normalized LVSW remains within $10 \%$ of maximal LVSW under basal hemodynamic conditions.

Effects of altered contractility on LV-arterial coupling relations. The effects of altered inotropy on hemodynamic parameters and $L V$-arterial coupling relations were also evaluated during a steady-state infusion of dobutamine in seven additional patients. These data are shown in Table II and are schematically demonstrated in Fig. 1. Dobutamine caused $\mathrm{E}_{\mathrm{es}}$ to increase from $2.71 \pm 1.07$ to $4.93 \pm 3.45 \mathrm{~mm} \mathrm{Hg} / \mathrm{ml}$ without a 


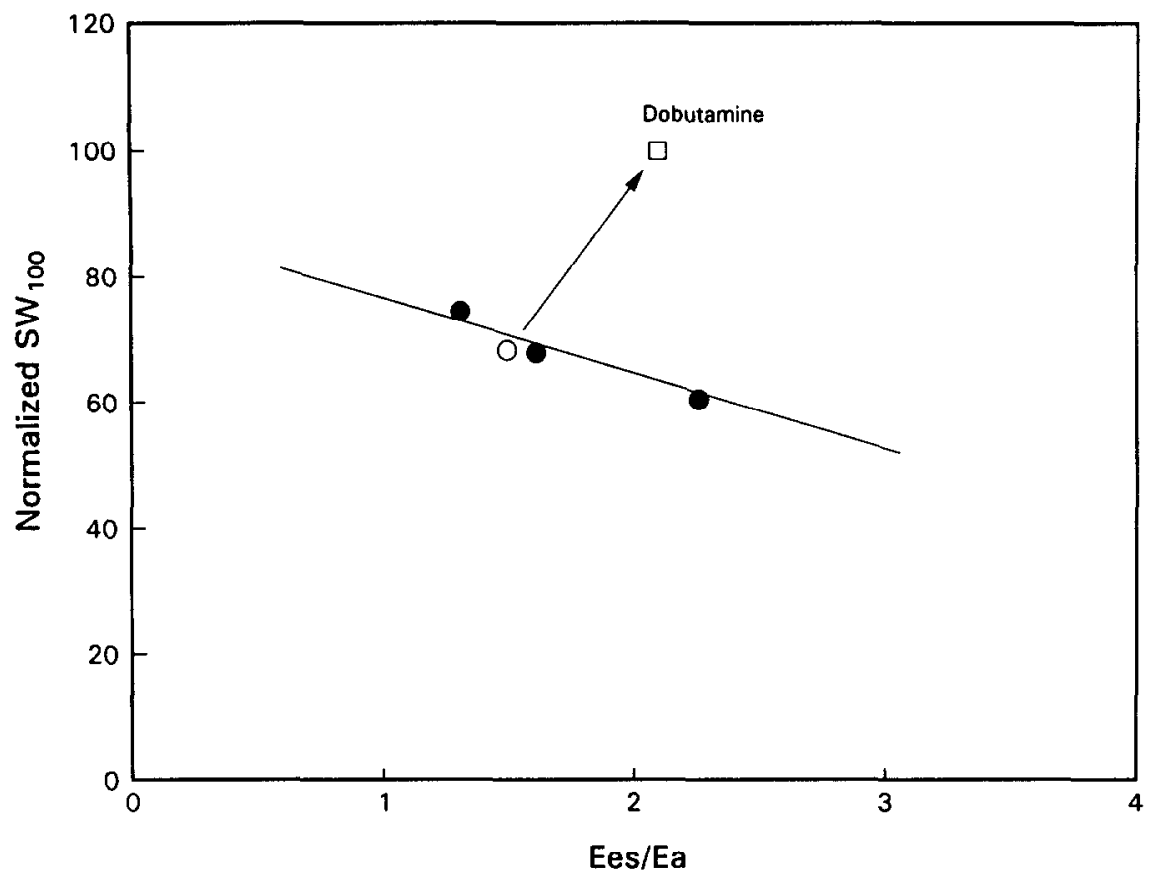

Fig. 3. $\mathrm{LV}$ stroke work $(S W)$ values standardized to a $\mathrm{LV}$ diastolic volume of $100 \mathrm{ml}$ have been normalized and plotted for each LV loading condition and inotropic state relative to the maximum LVSW (ordinate) and are compared with the $\mathrm{E}_{\mathrm{es}} / \mathrm{E}_{\mathrm{a}}$ ratios (abscissa). It is apparent that the normal $\mathrm{LV}$ operates over a hroad range of LVSW values under basal contractile conditions, while enhancement of the inotropic state increases the energy transfer from the LV to the arterial system for any value of $\mathrm{E}_{\mathrm{es}} / \mathrm{E}_{\mathrm{a}}$.

Table II. Effects of altered inotropy on hemodynamics and left ventricular-arterial coupling

\begin{tabular}{lcccccccccc}
\hline & $\begin{array}{c}E_{e s} \\
(\mathrm{~mm} \mathrm{Hg} / \mathrm{ml})\end{array}$ & $\begin{array}{c}V_{o} \\
(\mathrm{ml})\end{array}$ & $\begin{array}{c}P_{\text {es }} \\
(\mathrm{mm} \mathrm{Hg})\end{array}$ & $\begin{array}{c}V_{125} \\
(\mathrm{ml})\end{array}$ & $\begin{array}{c}E D V \\
(\mathrm{ml})\end{array}$ & $\begin{array}{c}S V \\
(\mathrm{ml})\end{array}$ & $\begin{array}{c}E_{a} \\
(\mathrm{~mm} \mathrm{Hg} / \mathrm{ml})\end{array}$ & $E_{e s} / E_{a}$ & $\begin{array}{c}S W_{100} \\
(g m-m)\end{array}$ & $\begin{array}{c}S W / P V A \\
\text { (gm }\end{array}$ \\
\hline Control & $2.71 \pm 1.07$ & $-9 \pm 14$ & $127 \pm 34$ & $43 \pm 16$ & $114 \pm 41$ & $68 \pm 26$ & $2.02 \pm 0.70$ & $1.48 \pm 0.66$ & $78 \pm 18$ & $0.68 \pm 0.11$ \\
Dobutamine & $4.93 \pm 3.45$ & $-7 \pm 17$ & $158 \pm 27^{*}$ & $28 \pm 11 \dagger$ & $97 \pm 27$ & $61 \pm 22$ & $2.92 \pm 1.23^{*}$ & $2.06 \pm 1.65$ & $114 \pm 27 \neq$ & $0.69 \pm 0.18$ \\
\hline
\end{tabular}

${ }^{*} p \leq 0.05, \dagger p<0.01, \neq p<0.001$ versus control.

$V_{125}$, Volume at a $\mathrm{P}_{\mathrm{es}}$ of $125 \mathrm{~mm} \mathrm{Hg}$; other abbreviations as in Table $\mathrm{I}$.

change in the volume-axis intercept $(-9 \pm 14$ vs $-7 \pm 17 \mathrm{ml})$. This caused the $\mathrm{V}_{125}$ at a $\mathrm{P}_{\mathrm{es}}$ of $125 \mathrm{~mm}$ $\mathrm{Hg}$ to decrease by $35 \%(p<0.01)$. Dobutamine also caused an increase in $P_{\text {es }}$ from $127 \pm 34$ to $158 \pm 27$ $\mathrm{mm} \mathrm{Hg}(p=0.05)$, while $L V \mathrm{~V}_{\mathrm{ed}}$ decreased insignificantly. $\mathrm{E}_{\mathrm{a}}$ increased from $2.02 \pm 0.70$ to $2.92 \pm 1.23$ $\mathrm{mm} \mathrm{Hg} / \mathrm{ml}(p<0.05)$, but this increase in $\mathrm{E}_{\mathrm{a}}$ was not as great as the increase in $\mathrm{E}_{\mathrm{es}}$. Consequently, $\mathrm{E}_{\mathrm{es}} / \mathrm{E}_{\mathrm{a}}$ also increased during the dobutamine infusion, from $1.48 \pm 0.66$ to $2.06 \pm 1.65$. Finally, LV stroke work standardized to a common $\mathrm{V}_{\mathrm{d}}$ of $100 \mathrm{ml}\left(\mathrm{SW}_{100}\right)$ increased from $78 \pm 18$ to $114 \pm 27 \mathrm{gm}-\mathrm{m}(p<0.001)$, but the SW/PVA ratio was unchanged by dobutamine.

As shown in Fig. 2, the relationship between LV SW/PVA and $\mathrm{E}_{\mathrm{es}} / \mathrm{E}_{\mathrm{a}}$ with dobutamine was similar to that under basal contractile conditions. In contrast, as shown in Fig. 3, the normalized LVSW is displaced upward for any comparable $\mathrm{E}_{\mathrm{es}} / \mathrm{E}_{\mathrm{a}}$ ratio. Thus, a greater degree of energy transfer from the LV to the arterial system can be performed at a comparable mechanical efficiency during an enhanced inotropic state.

\section{DISCUSSION}

LV performance must be evaluated in the context of its interaction with the systemic arterial system. Recent investigations by Sunagawa et al. ${ }^{1,2}$ have demonstrated, in the isolated left heart preparation, that a theoretic model of the interaction between $\mathrm{E}_{\mathrm{es}}$ and $\mathrm{E}_{\mathrm{a}}$ can be used to evaluate LV systolic pump function. They showed that when $E_{\text {es }}$ was matched with $E_{a}$, LVSW was optimized. A further theoretic investigation by Burkhoff and Sugawa ${ }^{3}$ demonstrated 
that over a range of contractile states, maximal LVSW occurred for each contractile state when $\mathrm{E}_{\mathrm{a}}$ matched $\mathrm{E}_{\mathrm{es}}$. This fulfilled the original observation of Sunagawa et al. ${ }^{1}$ They also demonstrated that myocardial efficiency, that is, the amount of external work performed for myocardial oxygen consumed, occurred during each contractile state when $\mathrm{E}_{\mathrm{a}}$ was approximately one-half $\mathrm{E}_{\mathrm{es}}$. Moreover, myocardial efficiency demonstrated a stronger dependence on $\mathrm{E}_{\mathrm{a}}$ than did LVSW during any stable contractile state. They, as did Sunagawa et al., 1, questioned whether it was possible to perform a physiologic amount of $\mathrm{LV}$ stroke volume at physiologic $L V V_{\text {ed }}$ and $P_{e s}$ when $E_{a}$ was equivalent to $\mathrm{E}_{\mathrm{es}}$. Both studies concluded that physiologic measures for these values would be more likely to occur when $\mathrm{E}_{\mathrm{a}}$ was approximately one-half $\mathrm{E}_{\mathrm{es}}$.

The present investigation was undertaken to determine how the normal human heart operated under basal hemodynamic conditions. The important observation of this investigation was that, in the normal human heart, LV chamber elastance and effective arterial elastance are coupled to operate over a broad range at a working point that was at neither optimum, although it more closely approximated maximal mechanical efficiency than maximal LVSW. When $\mathrm{E}_{\mathrm{a}}$ was increased using methoxamine, the $\mathrm{E}_{\mathrm{es}} / \mathrm{E}_{\mathrm{a}}$ ratio approached 1.0 . As a result, the LVSW increased compared with the control condition. In contrast, when $\mathrm{E}_{\mathrm{a}}$ was reduced by nitroprusside, there was a modest increase in the $\mathrm{E}_{\mathrm{es}} / \mathrm{E}_{\mathrm{a}}$ ratio at the expense of LVSW. However, when LVSW was normalized across the full range of $\mathrm{LV}$ loading conditions and the effects of preload on LVSW were considered, it was apparent that the normal human heart operated over a narrow range of LVSW values. At physiologic $L V V_{\text {ed }}$ and $\mathrm{P}_{\mathrm{es}}$, LVSW was within $10 \%$ of maximal LVSW.

The data in this investigation are consistent with several experimental observations in various animal preparations. Several animal studies have shown that LVSW or power are maximal when the LV and arterial input impedances are matched. Wilcken et al. ${ }^{4}$ have shown, in an open-chest canine preparation, that LVSW declines with changes in LV afterload. The observations made by Van den Horn et al., ${ }^{8}$ in an open-chest anesthetized cat preparation, also suggested that the LV operates to maximize LV output when physiologically loaded. This observation was carried further by Myhre et al. ${ }^{9}$ in an open-chest canine preparation, which showed that $\mathrm{SW}$ was matched to $\mathrm{LV}$ load. However, injection of microspheres into the left coronary circulation to produce LV dysfunction demonstrated that external output was less than maximum, suggesting an afterload mismatch when the LV was failing. Moreover, Elzinga et al., ${ }^{25}$ studying feline hearts on a Langendorf apparatus, showed that both the right and left ventricles were matched to the input impedances of their respective arterial systems, since both ventricles produced maximum power under normal physiologic loading conditions. In a closed-chest, conscious dog preparation, Little and Cheng ${ }^{11}$ demonstrated that LVSW was maximum when the $\mathrm{E}_{\mathrm{es}} / \mathrm{E}_{\mathrm{a}}$ ratio was nearly 1.0 .

Our data in the normal human heart also demonstrate that as the $\mathrm{E}_{\mathrm{es}} / \mathrm{E}_{\mathrm{a}}$ ratio approaches 1.0, LVSW increases toward a maximum. This was achieved at normal LV end-diastolic volumes and stroke volumes, but it required a $\mathrm{P}_{\mathrm{es}}$ value of $166 \mathrm{~mm} \mathrm{Hg}$, which was clearly in excess of what would be considered physiologic under basal hemodynamic conditions. Therefore, it would seem unlikely that the normal human heart operates to maximize LVSW under normal operating conditions. An $\mathrm{E}_{\mathrm{es}} / \mathrm{E}_{\mathrm{a}}$ ratio of 1.0 would be achieved only at nonphysiologic levels of LV load or possibly with LV dysfunction, which was not tested in this study. Nevertheless, these data also demonstrate that the normal human heart operates over a narrow range of LVSW values under basal hemodynamic conditions, suggesting a relative insensitivity of LVSW to LV load during basal contractile conditions.

Other studies have demonstrated that maximum LVSW or power do not coincide with optimal myocardial efficiency. Elzinga and Westerhof ${ }^{26}$ demonstrated, in an isolated physiologically ejecting feline heart preparation, that $\mathrm{LV}$ power was maximized at higher afterload resistance than that which optimized myocardial efficiency. Piene and Sund ${ }^{7}$ demonstrated that right ventricular pump function efficiency was maximal when pulmonary impedance and ejection from the right ventricle were in the physiologic range. Myhre et al. ${ }^{10}$ have also shown, in openchest anesthetized dogs, that the working point of the LV may not necessarily coincide with optimal LV external work. They suggested that under control conditions the LV and arterial load may not be optimally coupled, but the offset that they observed was too small to indicate whether the $\mathrm{LV}$ was controlled to maximize external work or efficiency. Finally, Burkhoff and Sagawa have shown that the optimal coupling between the LV and the systemic arterial system needed lo maximize LVSW occurs when the $\mathrm{LV}$ and arterial elastance values are matched, while maximal efficiency occurs when $\mathrm{E}_{\mathrm{a}}$ is approximatcly one-half $\mathrm{E}_{\mathrm{es}}$.

The average $\mathrm{E}_{\mathrm{es}} / \mathrm{E}_{\mathrm{a}}$ value of 1.62 under basal hemodynamic conditions in our patients was similar 
to that observed in a preliminary report by Jones et al. ${ }^{27}$ In nine isolated canine LV preparations, they reported that this $\mathrm{E}_{\mathrm{es}} / \mathrm{E}_{\mathrm{a}}$ ratio corresponded to maximal myocardial efficiency. When they normalized the myocardial efficiency values to a maximal myocardial efficiency value of 1.0 , a wide range of $\mathrm{E}_{\mathrm{es}} / \mathrm{E}_{\mathrm{a}}$ values around a normalized myocardial efficiency of 1.0 occurred, suggesting that the LV operates within $20 \%$ of this peak value. Recently, Asanoi et al. ${ }^{28} \mathrm{cal}-$ culated $\mathrm{E}_{\mathrm{es}}$ and $\mathrm{E}_{\mathrm{a}}$ in 12 normal patients using micromanometer LV pressures and M-mode echocardiograms. Interestingly, they noted that the $\mathrm{E}_{\mathrm{es}} / \mathrm{E}_{\mathrm{a}}$ ratio in these patients was approximately 2.0 . In the present investigation, it is important to note that a broad range of $\mathrm{E}_{\mathrm{es}} / \mathrm{E}_{\mathrm{a}}$ values exists at or near maximal mechanical efficiency. These data tend to confirm previous suggestions ${ }^{3}$ that for physiologic values of $P_{e s}, L V V_{e d}$, and LV stroke volume to occur, the $\mathrm{E}_{\mathrm{es}} / \mathrm{E}_{\mathrm{a}}$ ratio must reside somewhere above 1.0. Moreover, the relative sensitivity of LV mechanical efficiency to changes in loading conditions, in contrast to LV output, also suggests that the working point of the normal human heart may be regulated more by mechanical or myocardial efficiency than by maximal output or power.

The data in this investigation also demonstrate that LV mechanical efficiency is not reduced by an increase in LV contractility, despite enhanced energy transfer from the LV to the arterial system in the form of augmented normalized LVSW. This observation is consistent with the findings of previous clinical studies. ${ }^{29-31}$ These data suggest that the LV moves to a higher relationship between $\mathrm{SW}$ and $\mathrm{E}_{\mathrm{es}} /$ $\mathbf{E}_{\mathrm{a}}$, so that mechanical efficiency may be less sensitive to alterations in LV loading conditions.

Potential limitations to this investigation are based on the assumptions that were used. We did not estimate $\mathrm{MVO}_{2}$ and therefore we could not calculate myocardial efficiency. We assumed, however, a linear relationship between $\mathrm{MVO}_{2}$ and PVA. ${ }^{20,21}$ Despite this linear relationship, there is a $\mathrm{Y}$-axis offset to the $\mathrm{MVO}_{2} / \mathrm{PVA}$ relationship, which reflects a basal energy requirement, and therefore mechanical efficiency (SW/PVA) cannot be equated to myocardial efficiency $\left(\mathrm{SW} / \mathrm{MVO}_{2}\right)$. The average $\mathrm{E}_{\mathrm{es}} / \mathrm{E}_{\mathrm{a}}$ value of 1.62 under basal hemodynamic conditions in our patients and in the preliminary reports by Jones et al. ${ }^{27}$ lends support to the hypothesis that the normal human heart may have an operating point closer to maximal mechanical efficiency or myocardial efficiency under basal hemodynamic conditions then to maximal output. However, to prove this hypothesis, further investigations in patients with normal human hearts will be required.
We also assumed that $\mathrm{E}_{\mathrm{es}}$ was linear. However, $\mathrm{E}_{\mathrm{es}}$ may not be linear over the full range of LV loading conditions. ${ }^{32-34}$ This may be particularly true at the extremes of $L V$ load. It has been shown that a reduction in $\mathrm{LV}$ pressure and thus in coronary perfusion may cause this relationship to become curvilinear toward the volume axis. ${ }^{34}$ The observation that only a fraction of the potential energy stored within the LV at end systole can be converted to external work under an appropriate set of loading conditions because of shortening deactivation or cross-bridge interference at short sarcomere lengths, further suggests a curvilinear relationship. ${ }^{19}$ In addition, we assumed that $\mathrm{E}_{\mathrm{es}}$ was not affected by steady-state alterations in LV loading conditions. It has been previously shown that changes in LV loading conditions may cause a shift in the $\mathrm{E}_{\mathrm{es}}$ relationship. ${ }^{35}$

The final assumption of this investigation was the description of arterial load in terms of $E_{a}$. Recent preliminary information would suggest that utilization of $\mathbf{E}_{\mathrm{a}}$ to characterize arterial load is not unreasonable in the normal heart, since $\mathrm{E}_{\mathrm{a}}$ appears to reflect the major components of vascular load under normal conditions. ${ }^{36,37}$ It has been suggested that $E_{a}$ ignores the higher frequency components of arterial impedance because it assumes constancy of arterial pressure. The data of Latham et al. ${ }^{36}$ in the nonhuman primate and of Kelly et al. ${ }^{37}$ in hypertensive patients suggest that the use of $E_{a}$ to approximate total vascular load is appropriate. Thus, in the normal human heart, $\mathrm{E}_{\mathrm{a}}$ is probably a reasonable characterization of total vascular load, and it may be useful for characterizing total vascular load in patients with cardiac pathology to improve our understanding of $\mathrm{LV}$ and arterial interactions.

In conclusion, the data from this investigation suggest that under basal LV loading conditions, the interaction of the normal human heart with the systemic arterial system operates over a broad range, and the working point of the normal human heart is not at either optimum, neither maximal mechanical efficiency nor maximal LV output. The utility of this concept for clarifying the effects of pathologic conditions on the $\mathrm{LV}$-arterial coupling relation remains to be defined.

We thank Daniel G. Montgomery, BS, Christina Frank, BS, Janet Petrusha, RN, and Kim Arment for their assistance with the preparation of the manuscript.

\section{REFERENCES}

1. Sunagawa K, Maughan WL, Burkhoff D, Sagawa K. Left ventricular interaction with arterial load studied in isolated canine ventricle. Am J Physiol 1983;245:H773-80.

2. Sunagawa K, Maughan WL, Sagawa K. Optimal arterial 
resistance for the maximal stroke work studied in isolated ca nine left ventricle. Circ Res 1985;56:586-95.

3. Burkhoff D, Sagawa K. Ventricular efficiency predicted by an analytical model. Am J Physiol 1986;250:R1021-7.

4. Wilcken DEL, Charlier AA, Hoffman JIE, Guz A. Effects of alterations in aortic impedance on the performance of the ventricles. Circ Res 1964;14:283-93

5. Elzinga G, Westerhof N. Pressure and flow generated by the left ventricle against different impedances. Circ Res 1973; 32:178-86.

6. Piene H, Sund T. Flow and power output of right ventricle facing load with variable input impedance. Am J Physiol 1979;237:H125-30.

7. Piene $H$, Sund T. Does normal pulmonary impedance constitute the optimum load for the right ventricle? Am J Physiol 1982;242:H154-60

8. Van den Horn GJ, Westerhof N, Elzinga G. Optimal power generation by the left ventricle. Circ Res 1985;56:252-61.

9. Myhre ESP, Johansen A, Bjornstad J, Piene H. The effect of contractility and preload on matching between the canine left ventricle and afterload. Circulation 1986;73:161-71.

10. Myhre ESP, Johansen A, Piene H. Optimal matching between canine left ventricle and afterload. Am J Physiol 1988; 254:H1051-8.

11. Little WC, Cheng C. Left ventricular-arterial coupling in conscious dogs. Am J Physiol 1991;261:H70-6.

12. Starling MR, Montgomery DG, Mancini GBJ, Walsh RA Load independence of the rate of isovolumic relaxation in Inan. Circulation 1987;76:1274-81.

13. Starling MR, Montgomery DG, Walsh RA. Load dependence of the single beat maximal pressure (stress)-volume ratios in man. J Am Coll Cardiol 1989;14:345-53.

14. Starling MR, Gross MD, Walsh RA, Dell'Italia LJ, Montgomery DG, Squicciarini SA, Blumhardt R. Assessment of the ra dionuclide angiographic left ventricular maximum time-varying elastance calculation in man. J Nucl Med 1988;29:1368-81.

15. Starling MR, Dell'Italia LJ, Walsh RA, Little WC, Benedetto AR, Nusynowitz ML. Accurate estimates of absolute left ventricular volumes from equilibrium radionuclide angiographic count data using a simple geometric attenuation correction. $J$ Am Coll Cardiol 1984;3:789-98.

16. Starling MR, Dell'Italia LJ, Nusynowitz ML, Walsh RA, Little WC, Benedetto AR. Estimates of left ventricular volumes by equilibrium radionuclide angiography: importance of attenuation correction. J Nucl Med 1984;24:12-20.

17. Suga H. Total mechanical energy of a ventricle model and cardiac oxygen consumption. Am J Physiol 1979;236:H498 505.

18. Suga H, Hayashi T, Shirahata M. Ventricular systolic pressure-volume area as predictor of cardiac oxygen consumption. Am J Physiol 1981;240:H30-44.

19. Suga $H$. External mechanical work from relaxing ventricle. Am J Physiol 1979;236:H494-7.

20. Suga H, Hayashi T, Shirahata M, Suehiro S, Hisano R Regression of cardiac oxygen consumption of ventricular pressure-volume area in dog. Am J Physiol 1981;240:H320-5.

21. Starling MR, Mancini GBJ, Montgomery DG, Gross MD. Re lation between maximum time-varying elastance pressurevolume areas and myocardial oxygen consumption in dogs. Circulation 1991;83:304-14.
22. Little WC, Badke FR, O'Rourke RA. Effect of right ventriular pressure on the end-diastolic left ventricular pressure volume relationship before and after chronic right ventricular overload. Circ Res 1984:56:719 30.

23. Glower DD, Spratt JA, Snow ND, Kabas JS, Davis JW, Olsen CO, Tyson GS, Sabiston DC, Rankin JS. Linearity of the Frank-Starling relationship in the intact heart: the concept of preload recruitable stroke work. Circulation 1985;71:994-1009.

24. Little WC, Cheng CP, Mumma M, Igarashi Y, VintenJohansen $J$, Johnson WE. Comparison of measures of left ventricular contractile performance derived from pressurevolume loops in conscious dogs. Circulation 1989:80:1378-87.

25. Elzinga $G$, Piene $H$, De Jong JP. Left and right ventricular pump function and consequences of having two pumps in one heart. Circ Res 1980;46:564-74.

26. Elzinga, Westerhof N. Pump function of the feline left heart: changes with heart rate and its bearing on the energy balance. Cardiovas Res 1980;14:81-92.

27. Jones SR, de Tombe PP, Burkhoff D, Kass DA. Optimization of total ventricular efficiency studied in isolated canine hearts [Abstract]. Circulation 1990;82:1II-695.

28. Asanoi H, Sasayama S, Kameyama T. Ventriculo-arterial coupling in normal and failing heart in humans. Circ Res $1989 ; 65: 483-93$

29. Baxley WA, Dodge HT, Rackley CE, Sandler H, Pugh D. Left ventricular mechanical efficiency in man with heart disease. Circulation 1977;55:564-8.

30. Hasenfuss $\mathrm{G}$, Holubarsch $\mathrm{C}$, Heiss $\mathrm{HW}$, Meinertz T, Bonzel T, Waiss U, Lehmann M, Just $H$. Myocardial energetics in patients with dilated cardiomyopathy: influence of nitroprusside and enoximone. Circulation 1989:80:51-64

31. Kameyama 'I', Asanoi H, Ishizaka S, Yamanishi K, Fujita M, Sasayama S. Energy conversion efficiency in human left ventricle. Circulation 1992;85:988-96

32. Burkhoff D, Sugiura S, Yue DT, Sagawa K. Contractility-dependent curvilinearity of end-systolic pressure volume relations. Am J Physiol 1987;252:H1218-27.

33. Maughan WL, Sunagawa K, Burkhoff D, Sagawa K. Effect of arterial impedance changes on the end-systolic pressure-volume relation. Circ Res 1984;54:595-604.

34. Sunagawa K, Maughan WL, Fiesinger G, Guzman P, Chang MS, Sagawa K. Effects of coronary arterial pressure on left ventricular end-systolic pressure-volume relation of isolated canine heart. Circ Res 1982;50:727-34

35. Freeman GL, Little WC, O'Rourke RA. The effect of vasoactive agents on the left ventricular end-systolic pressurevolume relation in closed-chest dogs. Circulation 1986;74:110713

36. Latham RD, Rubal BJ, Spikema P, Westerhof N, Virmani R, Robinowitz M, Walsh RA. Ventricular/vascular coupling and regional arterial dynamics in the chronically hypertensive ba boon: correlation with cardiovascular structural adaption. Circulation 1988;63:798-811.

37. Kelly RP, Ting CT, Yang TM, Liu CP, Maughan WL, Chang MS, Kass DA. Similarity of arterial elastance estimates from ventricular and vascular parameters in man. Circulation $1992 ; 86: 513-21$ 